\title{
Determination of the Young's Modulus of a TiN Thin Film by Nanoindentation: Analytical Models and FEM Simulation*
}

\author{
Hélène Isselé \\ CEA Leti MINATEC Campus, 17 rue des Martyrs, 38054 Grenoble Cedex 9, France, \\ SIMaP, UMR 5266 CNRS/INPG/UJF, 1130 rue de la Piscine, \\ BP 7538402 Saint-Martin-d'Heres Cedex, France, and \\ MATEIS, 7 Avenue Jean Capelle, 69621 Villeurbanne Cedex, France \\ David Mercier \\ CEA Leti MINATEC Campus, 17 rue des Martyrs, 38054 Grenoble Cedex 9, France, and \\ SIMaP, UMR 5266 CNRS/INPG/UJF, 1130 rue de la Piscine, \\ BP 7538402 Saint-Martin-d'Heres Cedex, France \\ Guillaume Parry and Rafael Estevez \\ SIMaP, UMR 5266 CNRS/INPG/UJF, 1130 rue de la Piscine, \\ BP 7538402 Saint-Martin-d'Heres Cedex, France \\ Lionel Vignoud \\ CEA Leti MINATEC Campus, 17 rue des Martyrs, 38054 Grenoble Cedex 9, France \\ Christian Olagnon \\ MATEIS, 7 Avenue Jean Capelle, 69621 Villeurbanne Cedex, France \\ (Received 8 August 2012; Accepted 22 October 2012; Published 24 November 2012)
}

\begin{abstract}
The Young's modulus of a titanium nitride thin film has been investigated in this study by means of nanoindentation technique. Nanoindentation measurements have been coupled to analytical models and finite elements simulation to decorrelate the effect of substrate from the Young's modulus evaluation. Whereas analytical models did not lead to a converging solution towards experimental measurements, an identification based on finite elements calculations is shown necessary. Simulated contact stiffness versus contact areas have been fit with experiments and have led to a Young's modulus of $200 \pm 20 \mathrm{GPa}$ for a $180 \mathrm{~nm}$ thin film of TiN deposited on a $\langle 100\rangle$ silicon substrate. This study confirms the property of the TiN deposited in thin films to have a different Young's modulus from a bulk TiN. [DOI: 10.1380/ejssnt.2012.624]
\end{abstract}

Keywords: Titanium Nitride; Thin films; Nanoindentation; Finite element modelling; Analytical models; Young's modulus

\section{INTRODUCTION}

In microsystems and microelectronics, a reliable knowledge and estimate of the mechanical properties on layered structures with single or multiple thin film(s) is necessary to evaluate the stress distribution generated during their processing e.g. when materials with a mechanical mismatch are submitted to different thermal budgets. The properties of thin films are known to be noticeably different from those estimated in a bulk, as soon as the film thickness becomes smaller than $100 \mathrm{~nm}$.

Titanium nitride, widely used in microelectronics and microsystems as a protective layer and thus mainly deposited in thin films for this purpose, is known to exhibit this feature. Hence several studies have been reported in the literature to retrieve TiN's Young's modulus. It has been evaluated between 300 and $450 \mathrm{GPa}$ in a bulk $[1,2]$ (above $1 \mu \mathrm{m}$ thick), whereas a larger dispersion is found for lower thicknesses [3, 4], with a Young's modulus evaluated between 100 and $450 \mathrm{GPa}$, depending on thicknesses and process conditions [5]. Therefore, literature cannot be

* This paper was presented at the 16th International Conference on Solid Films and Surfaces (ICSFS-16), University of Genoa, Genoa, Italy, 1-6 July, 2012.

$\dagger$ Corresponding author: helene.issele@cea.fr

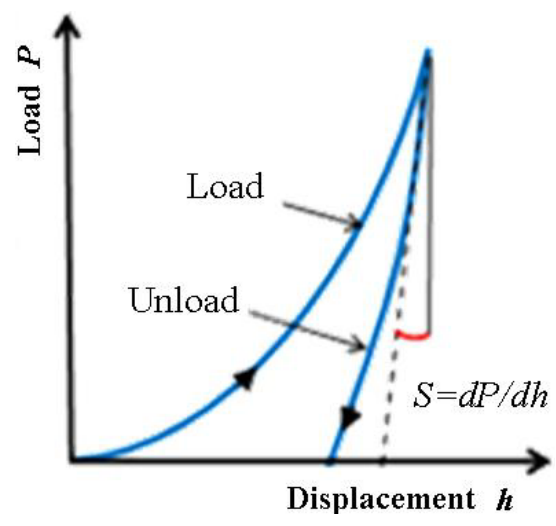

FIG. 1: Typical indentation curve obtained by loading and unloading the indenter's tip into a sample.

a reliable source if the same conditions and thicknesses as studied here are not reported, that is the reason why a specific characterization is necessary.

In this context, nanoindentation is useful to evaluate materials' Young's modulus. Figure 1 shows a typical curve obtained by the loading and unloading of the indenter into a sample.

From output measurements, i.e. the load $P$, the displacement $h$ and the contact stiffness $S$, i.e. the slope of 


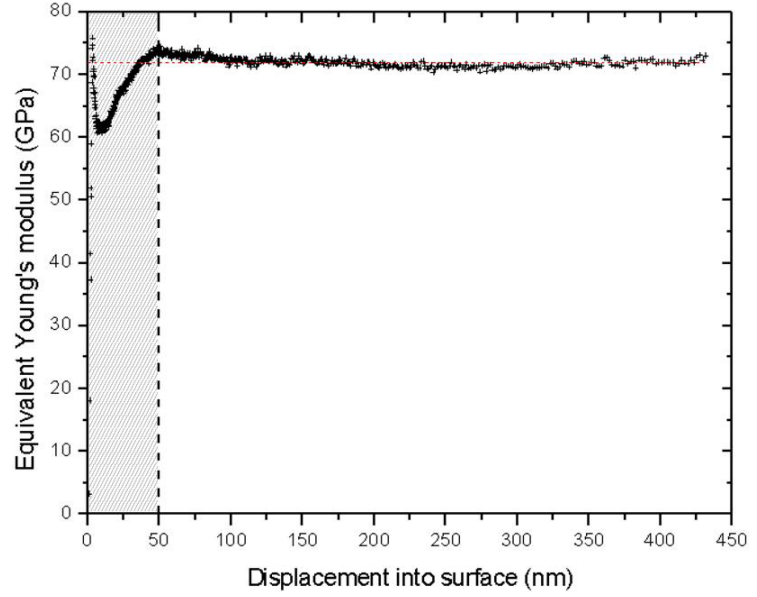

FIG. 2: Uncertainty in the Young's modulus evaluation for low indentation depths; example of the nanoindentation of the reference material : bulk fused silica $\left(E_{\text {eq }}^{\prime}=72 \mathrm{GPa}\right)$.
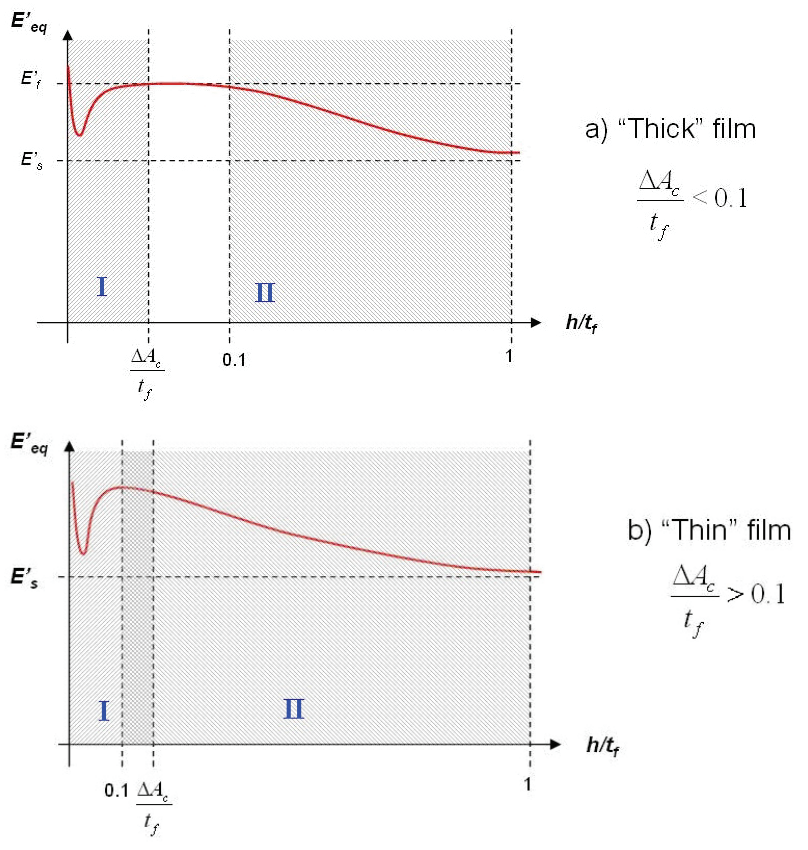

FIG. 3: Influence of the film thickness on the equivalent Young's modulus evaluation for two film/substrate configurations: thick (a) and thin (b) film on substrate.

the load-displacement curve at the beginning of the unloading, the expression (1) gives access to an equivalent reduced Young's modulus $E_{\text {eq }}^{\prime}$, depending on materials which interact during the test, the sample's and tip indenter's ones [6]:

$$
E_{\text {eq }}^{\prime}\left(E_{\text {ind }}^{\prime}, E_{\text {sam }}^{\prime}\right)=\frac{S}{2} \sqrt{\frac{\pi}{A_{\mathrm{c}}(P, h)}} .
$$

$E_{\text {sam }}^{\prime}$ and $E_{\text {ind }}^{\prime}$ are respectively the Young's moduli of the sample and the indenter, and $A_{\mathrm{c}}$ is the contact area, i.e. the projected area impacted by the indentation, depending on the load $P$ and the displacement $h$. The influence of the tip's elastic properties can be decorrelated from the sample's ones with the formula (2):

$$
\frac{1}{E_{\mathrm{eq}}^{\prime}}=\frac{1}{E_{\mathrm{sam}}^{\prime}} \frac{1}{E_{\mathrm{ind}}^{\prime}} .
$$

But when the sample is made of a film deposited on a substrate, it becomes difficult to retrieve the film Young's modulus directly from measurements. The Young's modulus of the sample is a combination of the Young's moduli of the thin film $\left(E_{\mathrm{f}}^{\prime}\right)$ and the substrate $\left(E_{\mathrm{s}}^{\prime}\right)[7]$.

Empirical methods are reported in the literature to get the film Young's modulus directly from experimental measurements. A rule says for example that below $10 \%$ of the film thickness, the substrate effect is negligible [6]. Moreover, if the displacement $\mathrm{h}$ is low, parameters such as the sample roughness, the bad-known tip geometry at its extremity or surface organic contamination, lead to a high uncertainty $\Delta A_{\mathrm{c}}$ in the contact area evaluation and so in the Young's modulus determination thanks to formula (1), as shown in Fig. 2 [6].

Therefore, for thin films it becomes difficult to trustfully evaluate the film Young's modulus. There is no range for indentation depths in which the substrate effect and the contact area evaluation uncertainty is negligible. Qualitatively, Fig. 3 presents the variation of $E_{\text {eq }}^{\prime}$ with the indentation depths for two cases of film/substrate configuration. Two areas are presented for both cases. The first area (I) corresponds to low indentation depths $\left(h / t_{\mathrm{f}}<\Delta A_{\mathrm{c}} / t_{\mathrm{f}}\right)$, for which the uncertainty in the contact area determination leads to unreliable Young's modulus evaluation. The second zone (II) is the range of indentation depths for which the substrate effect can no more be neglected (the $10 \%$ empiric rule, $h / t_{\mathrm{f}} \geq 0.1$ ). In the a) configuration, the film is thick enough so that the two regions do not overlap. The Young's modulus of the film can then easily be decorrelated from the substrate's one. In case b), as the film is thin, the two regions overlap; thus, no indentation depth can directly lead to a trustful evaluation of the film Young's modulus.

Knowing this, further analyses should be made to trustfully evaluate the thin film's Young modulus, using analytical models and/or numerical simulation. The goal of this study is to retrieve a thin film of TiN's Young's modulus by nanoindentation, and to compare values obtained using analytical models with those extracted from inverse modeling with the finite element method (FEM).

\section{EXPERIMENTAL}

\section{A. Sample preparation and indentation conditions}

The titanium nitride thin film was processed by physical vapor deposition, with a $12 \mathrm{~kW}$ plasma power and a process temperature of $350^{\circ} \mathrm{C} .180 \mathrm{~nm}$ were deposited on a $725 \mu \mathrm{m}$ thick $\langle 100\rangle$ silicon substrate. Figure 4 shows a SEM picture of the TiN thin film thus deposited.

The indentation tests were performed with a Nanoindenter XP MTS microprobe, using the Continuous Stiffness Measurement (CSM) mode. Four series of indents of $80 \mathrm{~nm}, 110 \mathrm{~nm}, 130 \mathrm{~nm}$ and $150 \mathrm{~nm}$ were performed on the sample, which represent respectively $45 \%, 60 \%, 72 \%$ and $83 \%$ of the film thickness. These depths were chosen 


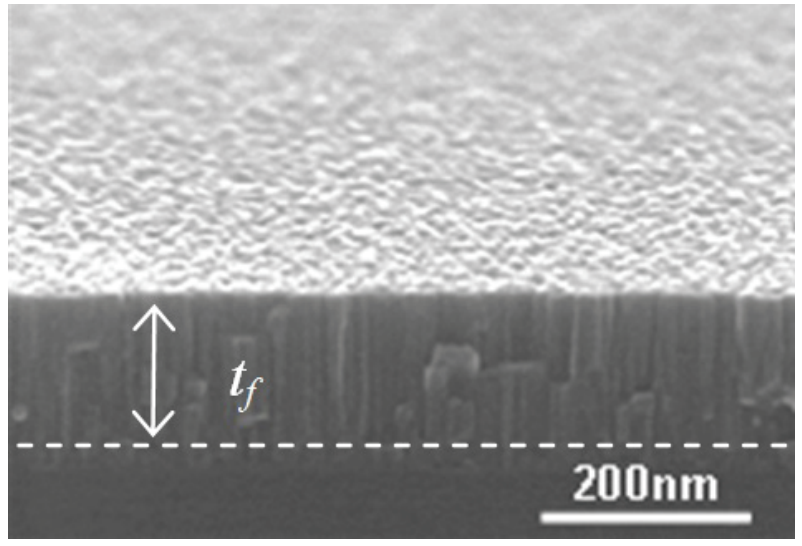

FIG. 4: SEM picture of the $180 \mathrm{~nm}$ thin film of TiN.

(a)

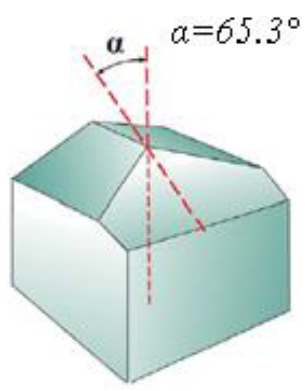

(b)

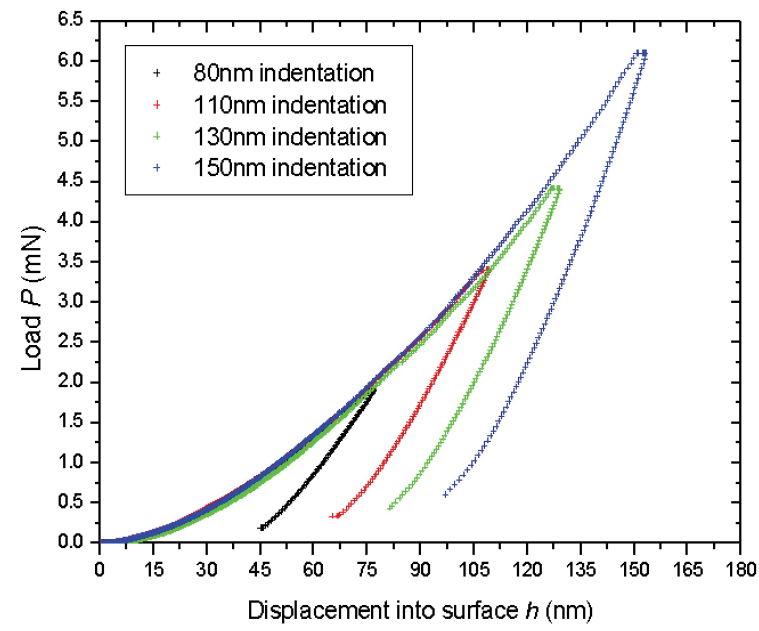

FIG. 5: Berkovich tip (a) and load-displacement curves (b) obtained for the four TiN's indentations.

to overcome the mentioned uncertainty $\Delta A_{\mathrm{c}}$ in the contact area evaluation, and to keep indentations into the film. The film was indented with a Berkovich tip, which is a three-sided pyramidal tip, as shown in Fig. 5(a). Figure 5(b) shows the experimental load-displacement curves obtained for mentioned depths of indentation with the Berkovich tip.

Two different methodologies are used to extract the Young's modulus of TiN from these experimental results.

\section{B. Experimental methodology}

The CSM mode gives access to the reduced Young's moduli $E_{\mathrm{sam}}^{\prime \text { exp }}$, calculated with the expressions (1) and (2), for each displacement $\mathrm{h}$ of the tip into the sample. These estimations of the Young's modulus will then be compared to those $E_{\text {sam }}^{\text {th }}$, derived from analytical models. These models determine $E_{\mathrm{sam}}^{\text {th }}$ with formulas depending on the film's $E_{\mathrm{f}}^{\prime}$ and substrate's $E_{\mathrm{s}}^{\prime}$ ones. Knowing the silicon elastic properties and fitting the experimental Young's modulus $E_{\text {sam }}^{\prime \text { exp }}$ with the theoretical one $E_{\text {sam }}^{\prime \text { th }}$, it is thus possible to retrieve the film's Young's modulus.

The other methodology to get a Young's modulus is to use measurements and finite element calculations. Reproducing the sample's mechanical behavior by FEM, it is possible to adjust the thin film's properties from the best fit between the Stiffness-contact area curves obtained in the simulations and the experimental measurements. In our study, the contact stiffness $S$ depends on the contact area $A_{\mathrm{c}} . S$ will then be compared in order to be able to adjust only the film's elastic properties, as explained in the next sections.

\section{RESULTS AND DISCUSSIONS}

\section{A. Analytical models}

Two different analytical models were considered to analyze experimental data. The first was proposed in 1992 by Gao et al. [8]. This model relies on finite element method to establish an analytical expression, based on a "weight function" $\Phi_{\mathrm{Gao}}$, of a combined Young's modulus $E_{\mathrm{sam}}^{\prime \text { Gao }}$, depending on the film's $E_{\mathrm{f}}^{\prime}$ and substrate's $E_{\mathrm{s}}^{\prime}$ ones, well captured by the formula (3):

$$
E_{\mathrm{sam}}^{\prime \mathrm{Gao}}=E_{\mathrm{s}}^{\prime}+\left(E_{\mathrm{s}}^{\prime}-E_{\mathrm{f}}^{\prime}\right) \Phi_{\mathrm{Gao}}\left(\frac{a}{t_{\mathrm{f}}}\right),
$$

where $t_{\mathrm{f}}$ and $a$ are respectively the film thickness and contact radius, and:

$$
\begin{aligned}
\Phi_{\mathrm{GaO}}(x)= & \frac{2}{\pi} \arctan \frac{1}{x}+\frac{1}{2 \pi\left(1-\nu_{\mathrm{f}}\right)} \times \\
& {\left[\left(1-2 \nu_{\mathrm{f}}\right) \frac{1}{x} \ln \left(1+x^{2}\right)-\frac{x}{1+x^{2}}\right] }
\end{aligned}
$$

The contact radius is calculated according to (5):

$$
a=\sqrt{\frac{C_{1} h_{\mathrm{c}}^{2}+C_{2} h_{\mathrm{c}}+C_{3} h_{\mathrm{c}}^{1 / 2}}{\pi}},
$$

where $C_{1}, C_{2}$ and $C_{3}$ are coefficients determined by the tip calibration on fused silica, and $h_{\mathrm{c}}$ is the contact height calculated by the Oliver and Pharr model [9]:

$$
h_{\mathrm{c}}=h-0.72 \frac{P}{S}
$$

The second model which was used to retrieve TiN's Young's modulus was proposed in 2006 by Bec et al. [10], 
TABLE I: Initial parameters for analytical models.

\begin{tabular}{cccc}
\hline \hline Material & $\begin{array}{c}\text { Young's modulus } \\
(\mathrm{GPa})\end{array}$ & Poisson's ratio & $\begin{array}{c}\text { Thickness } \\
(\mathrm{nm})\end{array}$ \\
\hline$\langle 100\rangle$ Silicon & 180 & 0.28 & - \\
TiN & $?$ & 0.25 & 180 \\
\hline \hline
\end{tabular}

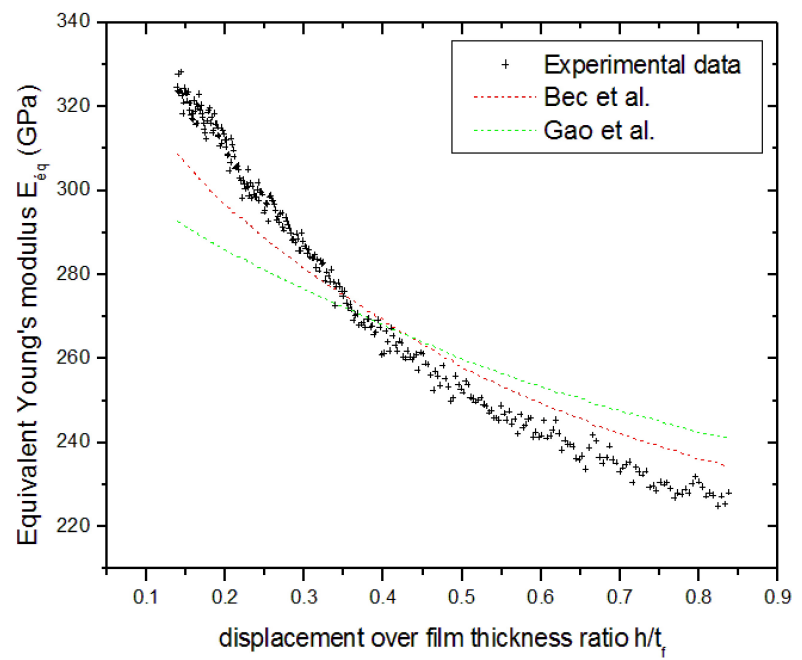

FIG. 6: Analytical results vs. experimental measurements.

and relies on analytical calculations based on the expression of the film and substrate stiffnesses associated in series, leading to formula (7):

$$
E_{\mathrm{sam}}^{\prime \mathrm{Bec}}=\left[\frac{2 a}{1+2 t_{\mathrm{f}} /(\pi a)}\left(\frac{t_{\mathrm{f}}}{\pi a^{2} E_{\mathrm{f}}^{\prime}}+\frac{1}{2 a e_{\mathrm{s}}^{\prime}}\right)\right]^{-1},
$$

where $t_{\mathrm{f}}$ and $a$ are respectively the film thickness and contact radius, calculated with the formula (5), the same way it has been calculated with Gao's model.

Initial parameters were fixed for both models and reported in Table I. Silicon elastic properties and TiN's Poisson ratio were taken from the literature [11, 12], whereas TiN's thickness was measured by the X-ray reflectivity technique.

For TiN, an approximate value of the Poisson ratio is enough for the indentation test as the reduced Young's Modulus $E^{\prime}$ is concerned. The parameter depends primary on the magnitude of the Young's modulus, and too little on the Poisson ratio that appears in the denominator with $E^{\prime}=E /\left(1-\nu^{2}\right)$.

Knowing these parameters, a least mean square method was applied on TiN's Young's modulus $E_{\mathrm{f}}^{\prime}$ to fit experimental reduced Young's modulus with theoretical ones, $E_{\text {Gao }}^{\prime}$ and $E_{\mathrm{Bec}}^{\prime}$. Figure 6 shows experimental measurements compared to theoretical calculations from the best fit obtained for both models. The best fits obtained for the Young's modulus of the TiN are reported in Table II.

Two points have to be noticed here: first, for both models there is not a good fit on the whole range of experimental data (see Fig. 6) when a single Young's modulus of the TiN is used. These fits lead to a large discrepancy in the identified Young's modulus, so that these analytical
TABLE II: Analytical best fits for the Young's modulus of TiN.

\begin{tabular}{cc}
\hline \hline Model & Best fit TiN's Young's modulus (GPa) \\
\hline Bec et al. & 800 \\
Gao et al. & 330 \\
\hline \hline
\end{tabular}
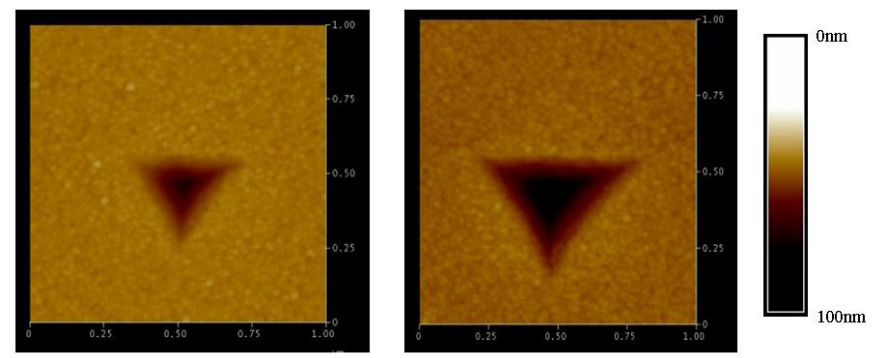

FIG. 7: $1 \times 1 \mu \mathrm{m}^{2}$ AFM image of the $80 \mathrm{~nm}$ (left) and $110 \mathrm{~nm}$ (right) indents.

models are not reliable in the configuration under consideration. This observation has motivated the development of a detailed finite element investigation of this nanoindentation test. This appears necessary to derive a reliable estimation of the Young's modulus of our specific thin film of TiN.

\section{B. Finite element simulations}

A specific investigation has been here developed to use finite element analysis to retrieve TiN's Young's modulus. A direct comparison between the load-depth experimental curve and FE calculations is not straightforward, as new inputs are necessary as the film's yield stress and hardening response that are known to be length-scale dependent and not easy to evaluate experimentally. In order to overcome this difficulty, we choose to compare the contact stiffness (see Eq. (1)) from the FE calculations with that measured experimentally. This approach allows considering an elastic problem that enables the identification of TiN's Young's Modulus (the Poisson ratio being fixed already).

This approach is motivated by the elastic sample response during the unloading, that provides a unique mechanical information depending on its elastic properties. The contact stiffness, defined by the slope of the loaddepth curve at the beginning of the unloading (see Fig. 1), versus the contact area is used for the identification. Practically, one performs indentations tests at different penetration depths. By acquiring an image of the indent (Fig. 7), and measuring the experimental contact area (with an image treatment software), the contact stiffness is derived from this measurement by the corresponding load level at the onset of unloading. This results in experimental data $S\left(A_{\mathrm{c}}\right)^{\exp }$ corresponding to specific contact areas that will be compared to the FE predictions.

In Table III, four measurements are reported that will be used for the FE identification of the Young's modu- 
(a)

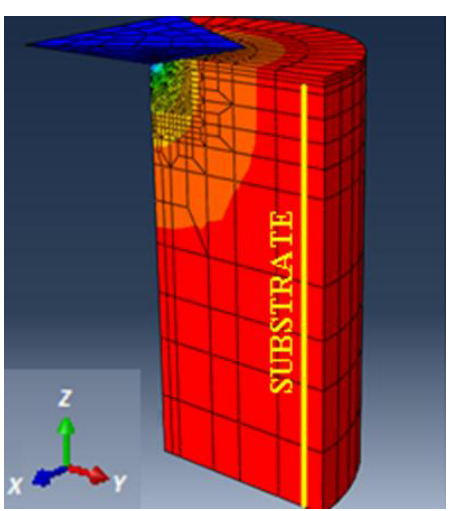

(b)

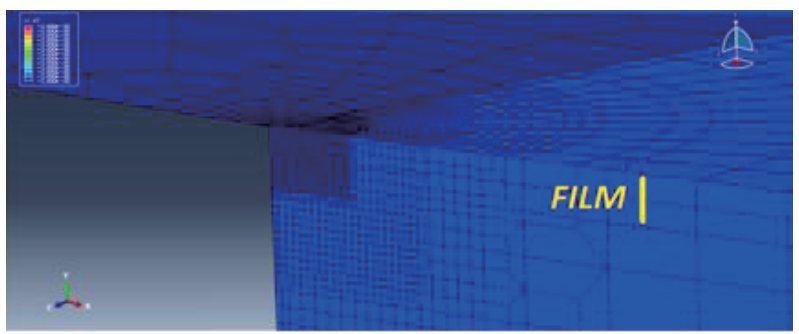

(c)

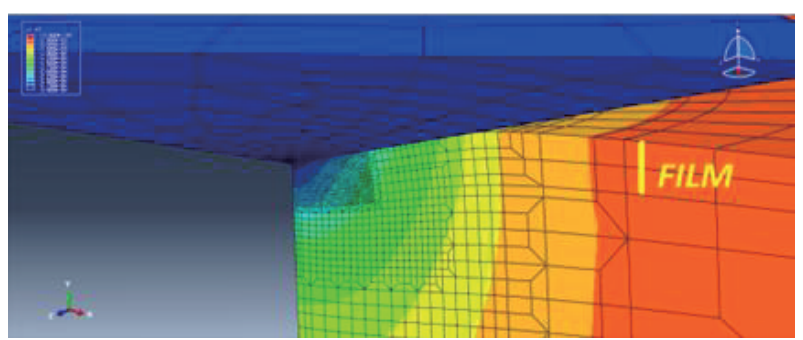

FIG. 8: Finite element model used in nanoindentation simulation: (a) whole sample after loading and (b) zoom before and (c) after indentation.

TABLE III: Experimental $S\left(A_{\mathrm{c}}\right)$ points.

\begin{tabular}{ccc}
\hline $\begin{array}{c}\text { Indentation height } \\
(\mathrm{nm})\end{array}$ & $\begin{array}{c}\text { Contact Stiffness } \\
(\mathrm{N} / \mathrm{m})\end{array}$ & $\begin{array}{c}\text { Contact Area } \\
\left(\mathrm{nm}^{2}\right)\end{array}$ \\
\hline 80 & 66600 & 63800 \\
110 & 86900 & 111100 \\
130 & 110100 & 193200 \\
150 & 132000 & 301000 \\
\hline \hline
\end{tabular}

lus to be presented next. To compare these experimental points with $\mathrm{FE}$ simulations, the $\mathrm{FE}$ software Abaqus ${ }^{\mathrm{TM}}$ is used with the isotropic-elastic properties of each constituent reported in Table IV. Because of the Berkovich tip geometry, it is possible to consider only one-third of the sample. A hard contact is assumed between the indenter and the film with no friction. A surface to surface interaction is used with Abaqus ${ }^{\mathrm{TM}}$ to handle practically the contact problem.

Boundary conditions are prescribed along the symmetry planes of the sample (substrate + film) and indenter to ensure the one-third geometry of the real problem: no displacement on lateral surface's normal direction, no (a)

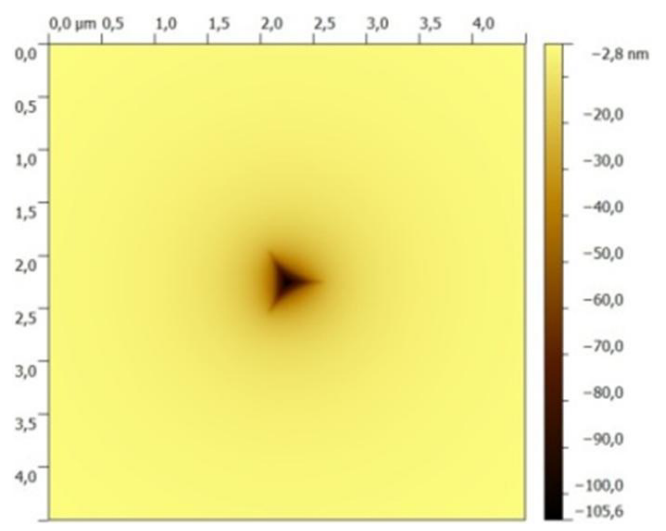

(b)

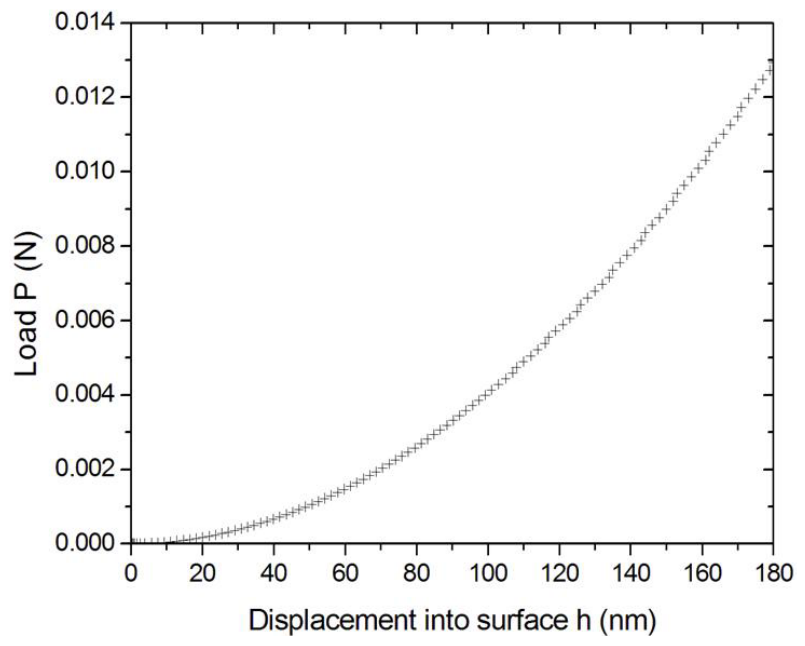

FIG. 9: Simulated load-displacement curve (left) and picture of a simulated indent (right).

displacement in the $Z$-direction for nodes located on the bottom face (the region of the substrate far from the contact region), and only $Z$ direction allowed for the central ridge's ones. The Berkovich tip was built with full account of its geometry. As the sample is totally elastic, loading or unloading the tip leads to the same mechanical behavior. It is thus sufficient only to simulate the loading of the tip into the sample; this is why the load step consists in final $180 \mathrm{~nm}$ tip displacement in the $Z$-direction, with no displacement in the other directions, thus covering the indentation depths reported experimentally (see Table III). The sample was meshed so that a finer mesh has been set around the indentation in the film, as shown in Fig. 8.

To get the simulated contact stiffness, the displacement and reaction force in the $Z$-direction of a reference point on the tip has been extracted. In parallel, displacement in the $Z$-direction and $(X, Y)$ coordinates of the top surface's nodes were extracted and treated to get an image of the indent during each step of the loading, as shown in Fig. 9.

Three different TiN's Young's modulus have been simulated, and the best fit compared to experimental points was obtained for a Young's modulus of $200 \pm 20 \mathrm{GPa}$, as shown in Fig. 10.

We showed in the first part that Bec's and Gao's ana- 
TABLE IV: Conditions for finite element simulations.

\begin{tabular}{|c|c|c|c|c|}
\hline \multicolumn{2}{|l|}{ System } & \multicolumn{3}{|c|}{ Properties $(E(\mathrm{GPa}) ; \nu)$} \\
\hline $\begin{array}{c}\text { Sample } \\
3 \text { D deformable } 120^{\circ} \text { symmetric }\end{array}$ & $\begin{array}{l}\text { Indenter tip } \\
\text { Rigid }\end{array}$ & $\begin{array}{l}\text { Si substrate } \\
(180 ; 0.28)\end{array}$ & $\begin{array}{l}\text { TiN thin film } \\
\left(E_{\mathrm{f}} ; 0.25\right) \\
\text { Isotropic Elastic }\end{array}$ & $\begin{array}{l}\text { Diamond tip [12] } \\
\quad(1017 ; 0.07)\end{array}$ \\
\hline
\end{tabular}

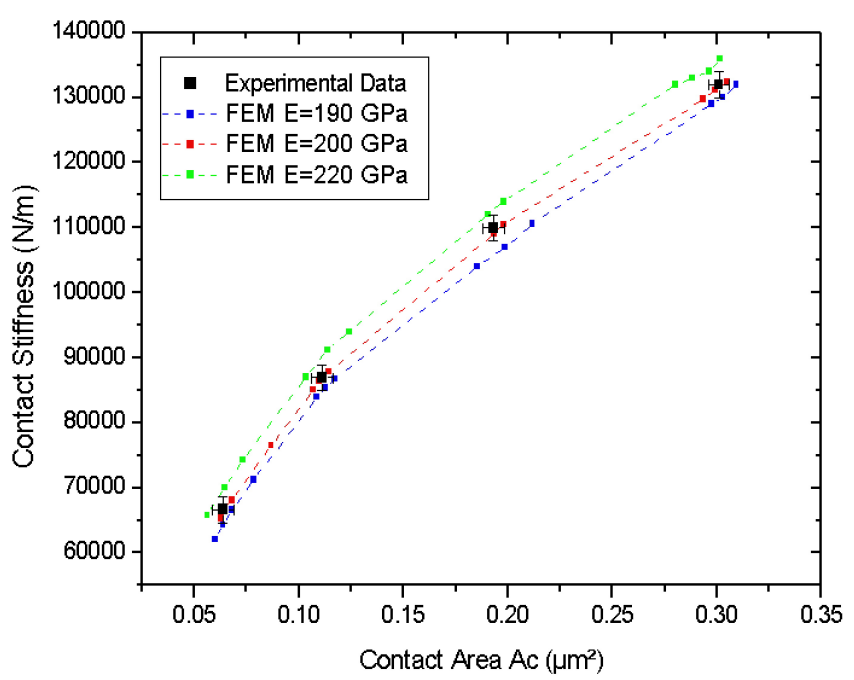

FIG. 10: FEM analyses fits versus experimental points.

lytical models led to a large dispersion in the TiN Young's modulus evaluation, ranging it between 300 and 800 GPa. These models are thus not reliable for our sample.

To explain such results, Perriot and Barthel [13] pointed out that many analytical models such as Bec's and Gao's ones rely on the Hertz contact theory [14] for homogeneous systems. This theory assumes that the indentation depth $h$ is directly proportional to the contact radius a, thus leading to Oliver and Pharr expression (7) to calculate the actual contact indentation depth $h_{\mathrm{c}}$. This expression becomes questionable when it comes to coated systems, and even more to thin films/substrate configurations.

Thus, full 3D finite element calculations were performed, which satisfy equilibrium conditions, leading to a TiN Young's modulus evaluated at 200土20 GPa. As long as no analytical model is available for this thin film configuration, finite elements calculations are required.

\section{CONCLUSIONS}

In this study, two analysis were applied to determine the Young's modulus by nanoindentation of a $180 \mathrm{~nm}$ thin film of titanium nitride deposited on a $\langle 100\rangle$ silicon substrate. The analytical models of Bec et al. and Gao et al. have been applied, but have led to a discrepancy in identified Young's moduli and a bad fit with experimental measurements. A specific investigation has thus been carried out using finite element analysis in order to have the TiN's Young's modulus as the only adjustable parameter to fit the experimental results. From this methodology, the contact stiffness versus the contact area was compared between the experimental measurements and the numerical simulations. This method allows deriving a reliable estimation for a TiN's Young's modulus of 200 \pm 20 GPa. These results confirm the particularity of the TiN to have some variable elastic properties for thin film deposits, compared to literature bulk's Young's modulus evaluated between 300 and $450 \mathrm{GPa}$.

These results could be further compared with another technique which is dedicated to thin film measurements: the picosecond acoustic technique.

\section{Acknowledgments}

The authors wish to thank $\mathrm{PhD}$ S. V. Hoang for his help in the finite element calculations, $\mathrm{PhD} \mathrm{M}$. Verdier for fruitful discussions and the LCFC lab for its help in AFM characterization.
[1] R. Ahuja, J. Wills, and B. Johansson, Phys. Rev. B 53, 3072 (1996).

[2] W. Meng and G. Eesley, Thin Solid Films 271, 108 (1995).

[3] J. Kuang and H. Chien, Adv. Sc. Lett. 4, 3570 (2011).

[4] M. Lichinchi, C. Lenardi, J. Haupt, and R. Vitali, Thin Solid Films 333, 278 (1998).

[5] P. Patsalas, C. Charitidis and S. Logothetidis, Surf. Coating Technol. 125, 335 (2000).

[6] A. Fischer-Cripps, Nanoindentation, Second edition (Springer, New York, 2004).

[7] J. Mencik, D. Munz, E. Quandt, E. R. Weppelmann, and M. V. Swain, J. Mater. Res. 12, 2475 (1997).

[8] H. Gao, C. Chu, and J. Lee, Int. J. Sol. Struct. 28, 2471
(1992).

[9] W. Oliver and G. Pharr, J. Mater. Res. 19, 3 (2004).

[10] S. Bec, A. Tonck, and J. Loubet, Phil. Mag. 28, 5347 (2006).

[11] M. Hopcroft, W. Nix, and T. Kenny, J. MEMS 19, 229 (2010).

[12] Y. Sun, A. Bloyce, and T. Bell, Thin Solid Films 271, 122 (1995).

[13] A. Perriot and E. Barthel, J. Mater. Res. 19, 600 (2004).

[14] H. Hertz, J. Reine und Angewandte Mathematik 92, 156 (1882). 